\title{
Nonclassical Optical Bistability and Resonance-Locked Regime of Photon- Pair Sources Using Silicon Microring Resonator
}

Guo, Kai; Yang, Lin; Shi, Xiaodong; Liu, Xuanming; Cao, Yining; Zhang, Jingjing; Wang, Xiaolin; Yang, Junbo; Ou, Haiyan; Zhao, Yijun

Published in:

Physical Review Applied

Link to article, DOI:

10.1103/PhysRevApplied.11.034007

Publication date:

2019

Document Version

Publisher's PDF, also known as Version of record

Link back to DTU Orbit

Citation (APA):

Guo, K., Yang, L., Shi, X., Liu, X., Cao, Y., Zhang, J., Wang, X., Yang, J., Ou, H., \& Zhao, Y. (2019).

Nonclassical Optical Bistability and Resonance-Locked Regime of Photon- Pair Sources Using Silicon Microring Resonator. Physical Review Applied, 11(3), [034007]. https://doi.org/10.1103/PhysRevApplied.11.034007

\section{General rights}

Copyright and moral rights for the publications made accessible in the public portal are retained by the authors and/or other copyright owners and it is a condition of accessing publications that users recognise and abide by the legal requirements associated with these rights.

- Users may download and print one copy of any publication from the public portal for the purpose of private study or research.

- You may not further distribute the material or use it for any profit-making activity or commercial gain

- You may freely distribute the URL identifying the publication in the public portal 


\title{
Nonclassical Optical Bistability and Resonance-Locked Regime of Photon-Pair Sources Using Silicon Microring Resonator
}

\author{
Kai Guo,,${ }^{1,}$ Lin Yang,,${ }^{1} \dagger$ Xiaodong Shi, ${ }^{2}$ Xuanming Liu, ${ }^{1}$ Yining Cao, ${ }^{1}$ Jingjing Zhang, ${ }^{3}$ \\ Xiaolin Wang, ${ }^{4}$ Junbo Yang, ${ }^{3}$ Haiyan Ou, ${ }^{2}$ and Yijun $Z_{h a o}{ }^{4}$ \\ ${ }^{1}$ Institute of Systems Engineering, AMS, Beijing 100039, China \\ ${ }^{2}$ Department of Photonics Engineering, Technical University of Denmark, Kongens Lyngby 2800, Denmark \\ ${ }^{3}$ College of Liberal Arts and Sciences, National University of Defence Technology, Changsha 410073, China \\ ${ }^{4}$ College of Advanced Interdisciplinary Studies, National University of Defence Technology, Changsha 410073, \\ China
}

(Received 20 November 2018; revised manuscript received 5 January 2019; published 4 March 2019)

\begin{abstract}
We generate correlated photon pairs in an all-pass silicon microring resonator using continuous-wave pumped spontaneous four-wave mixing and we characterize in detail the optical bistability in the nonclassical single-photon regime. Special attention is given to the resonance-locked method by balancing the pump-induced heating and the active off-chip cooling, leading to stable operation of the photon-pair source. The maximal coincidence rate of $20.53 \pm 0.34 \mathrm{~Hz}$, maximal coincidence-to-accidental ratio of $654 \pm 125$, and minimal zero-delayed heralded second-order correlation of $0.14 \pm 0.09$ are achieved. The wavelengths of photon pairs remain unchanged at different pump power, indicating that our source is more compatible with the common communication systems than those using conventional on-resonance strategies, that tunes the pump wavelength. This work not only experimentally validates the nonclassical bistability behaviors, but also puts forward a simple method of pump-resonance matching for high-quality performance.
\end{abstract}

DOI: 10.1103/PhysRevApplied.11.034007

\section{INTRODUCTION}

Correlated photon-pair sources have been widely studied in emergent applications of quantum optics, such as quantum computing [1] and quantum communication [2]. The most widely investigated technology in this field is the quantum key distribution [3-5], in which some schemes involve the nature of correlated photon pairs, such that the detection of one photon heralds the existence of the other. Sources capable of these schemes need to emit photon pairs in a probabilistic manner with high brightness and low noise, preferably operating at telecom wavelengths that fit the high transmittance window of the optical fiber networks. In the past decades, photon-pair sources have been achieved through a variety of spontaneous nonlinear processes, including the spontaneous parametric down-conversion in optical crystals [6] and quasimatched waveguides [7], and the spontaneous fourwave mixing (SpFWM) in highly nonlinear fibers [8-11] and integrated waveguides [12-20]. Although the fiberbased source via SpFWM is more compatible with the optical fiber networks than the source via spontaneous

*guokai07203@hotmail.com

†yanglin61s@126.com parametric down-conversion, it suffers from the huge noise induced by the spontaneous Raman scattering of amorphous silica at room temperature [9]. On the other hand, a number of photon-pair generation experiments have been carried out in on-chip platforms, due to the advantages of designable dispersion and highly nonlinear response. The materials of these platforms include crystalline silicon [12-16], amorphous silicon [17], silica [18], silicon nitride [19], and (Al,Ga)As [20].

It is appealing to integrate significant building blocks for quantum communication on a single chip, the functionalities of which include photon-pair generation, photonpair modulation, cryptogram coding, and cryptogram decoding [21,22]. Therefore, silicon-on-insulator (SOI) platforms are primarily concerned because of the compatibility with complementary metal-oxide-semiconductor technology that enables compact integration of electrically and optically driven components and the mature fabrication procedures that enable reproducible and reliable features for optical signal processing [23,24]. Moreover, as the Raman response of crystalline silicon has a narrow linewidth peak locating at a frequency detuning of $15.6 \mathrm{THz}$, the SOI platforms can get rid of the Raman-induced noise by easy filtering [25]. Additionally, through either butt-coupled approaches (e.g., 
plasma-cladded inverse tapers [26]) or vertical-coupled approaches [e.g., photonic-crystal-based grating couplers (PCGCs) [27] ], the SOI platforms can be efficiently connected to the optical fibers, which benefits photon-pair sources from both the highly nonlinear response of SOI waveguides, as the SpFWM medium, and the efficient noise suppression of fiber-based filters, resulting in ultrahigh coincidence-to-accidental ratio (CAR) [16].

Although photon-pair sources are often driven by pulsed pumps, continuous-wave lasers with merits of cheaper and easier on-chip interaction are required in special applications, such as time-energy entanglement [5,28-31], quantum wavelength division multiplexing [32-34], and covert quantum communication [35], because the narrow linewidth pump gives rise to a strong spectral anticorrelation of photon pairs [36-38]. On the other hand, to satisfy other applications that require uncorrelated photon pairs, such a spectral anticorrelation can be partially avoided by fiber-based filters. However, such a filtering strategy results in a relatively low coincidence rate and low pair brightness [16]. A promising solution is to use the microring resonator (MRR), which enables on-chip filtering and nonlinear enhancement simultaneously within a narrow bandwidth. Furthermore, photon-pair sources using MRR often require lower pump power for SpFWM, but they generate almost the same amount of photon pairs compared to that using straight waveguides and result in a pair brightness of several orders of magnitude higher within a small footprint, due to the narrow bandwidth [3951]. However, the loss-induced heat changes the refractive index and makes the resonance wavelength power dependent, which becomes stronger in SOI MRRs, due to nonlinear loss including two-photon absorption and free carrier absorption [52,53]. The power-dependent property, often named the thermal-based optical bistability [54-60], poses a challenge to keep the pump of photon-pair sources operating exactly at the on-resonance. Hence, both a solid understanding of the optical bistability in the nonclassical single-photon regime and an efficient method to achieve the resonance-locked regime are important.

In this paper, we study the optical bistability with respect to the pair rate of an all-pass SOI-MRR-based source using an off-chip temperature-controlling module and present an experimental validation. We propose a method to achieve a photon-pair source working in the resonance-locked regime by balancing the heat accumulation induced by the pump and heat dissipation facilitated by active off-chip cooling, which results in a stable and high-quality performance of the source. Such a method enables a photon-pair source that can be driven by a wavelength-fixed pump and emit photons efficiently with an unchanged wavelength at different power levels. Therefore, the proposed source becomes very compatible with the wavelength division multiplexing systems with no need for concomitant tuning. Detailed descriptions of experiments and systems are presented, which have usually been put briefly in previous works announcing that the MRR has to be thermally stabilized. Hence, this work is also easily followed with future investigations, where the stable on-resonance of the microcavity is desired.

\section{EXPERIMENTAL SETUP}

A schematic experimental setup is shown in Fig. 1, including the pair source, collection scheme, and detection scheme, corresponding to the measurements of the pair rate, coincidence rate, CAR, and heralded second-order correlation. Specifically, a continuous-wave laser, centered at $1549.78 \mathrm{~nm}$ and power-amplified to $25 \mathrm{dBm}$ by an erbium-doped fiber amplifier (EDFA), is utilized to drive the photon-pair source. Tunable band-pass filters centered at pump wavelength (PTBPFs) suppress the incident sideband noise, so that the signal-to-noise ratio at a wavelength detuning of $\pm 5 \mathrm{~nm}$ reaches $135 \mathrm{~dB}$. The incident power is adjusted by a tunable attenuator (ATT) ensuring a constant signal-to-noise ratio, while the incident polarization is adjusted by a polarization controller (PC) ensuring a minimal coupling loss through PCGCs $(12 \mathrm{~dB})$. After the SOI chip, $1 \%$ of the leaked pump field is power-monitored by a powermeter (PM), while the rest is suppressed by fiber Bragg gratings centred at pump wavelength (PFBGs) with an extinction ratio of $108 \mathrm{~dB}$. The remaining photons are separated into two arms by frequency using a circulator, a fiber Bragg grating centered at signal wavelength (SFBG), and a tunable band-pass filter centered at idler wavelength (ITBPF), then detected by avalanche single-photon detectors (SPDs, ID230, IDQ) and counted by a timetagger (ID801, IDQ). The collection efficiency and the deadtime of all SPDs are set at $20 \%$ and $10 \mu \mathrm{s}$, respectively, resulting in an average dark count rate of $75 \mathrm{~Hz}$.

The SOI chip is up-cladded by $1-\mu \mathrm{m}$-thick silica, where both the bus waveguide and the ring cavity of the investigated MRR have cross-sectional dimensions of $H \times W=$ $250 \times 450 \mathrm{~nm}$. The bus waveguide length of $1 \mathrm{~mm}$ (tapers included) ensures negligible SpFWM contribution, while the ring cavity radius of $100 \mu \mathrm{m}$ results in a free spectral range of $0.88 \mathrm{~nm}$ [see Fig. 1(d)]. Matching the transmittance windows of SFBG and ITBPF, only the photons from the fifth resonances on both sides of pump (signal at $1545.33 \mathrm{~nm}$ and idler at $1554.25 \mathrm{~nm}$ ) are detected. The full width at half maximum (FWHM) of the resonance at the pump wavelength is $\Delta \lambda=26 \mathrm{pm}$, and its quality factor [61]

$$
Q=\frac{\lambda_{\text {res }}}{\Delta \lambda}
$$

reaches $5.96 \times 10^{4}$. The SOI chip is placed on top of a copper terrace, whose temperature (defined as the external temperature) is measured by a thermal sensor, and it is controlled by a thermistor with a circuit, constituting the 


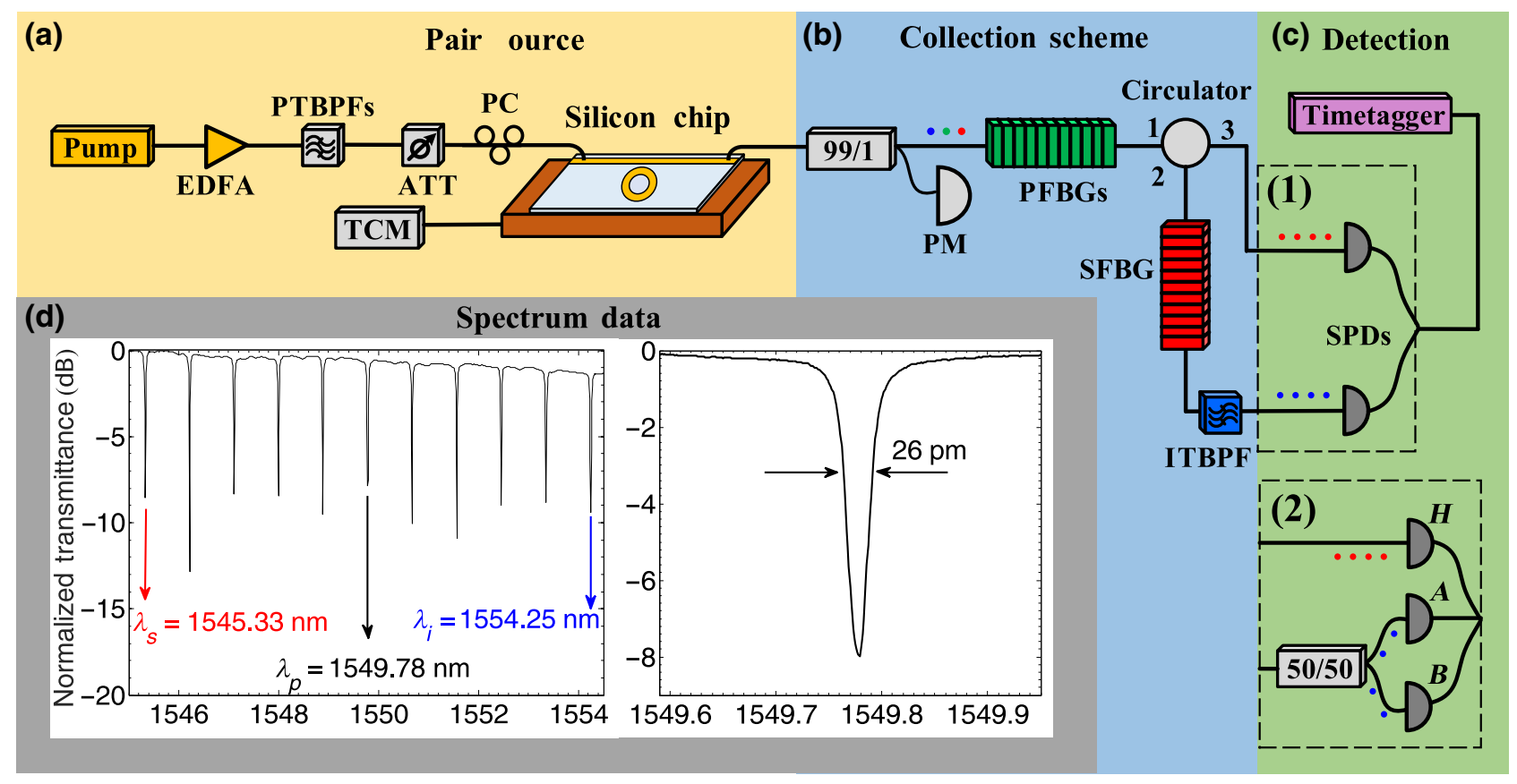

FIG. 1. Schematic experimental setup: (a) pair source, (b) collection scheme, (c) detection for (1) pair rate, coincidence rate, coincidence-to-accidental ratio and (2) heralded second-order correlation. EDFA denotes the erbium-doped fiber amplifier; TBPF, tunable band-pass filter; ATT, tunable attenuator; PC, polarization controller; TCM, temperature-controlling module; FBG, fiber Bragg grating; and SPD, single-photon detector. (d) The transmittance of the investigated MRR: the pump, signal, and idler wavelengths are at $1549.78,1545.33$, and $1554.25 \mathrm{~nm}$, respectively. The FWHM of the resonance corresponding to the pump is $26 \mathrm{pm}$.

temperature-controlling module. The room temperature is kept at $22^{\circ} \mathrm{C}$, where the initial wavelength of the resonance corresponding to the pump is $1550.5 \mathrm{~nm}$ (measured at $1 \mu \mathrm{W}$ ), and it reaches $1549.78 \mathrm{~nm}$ by cooling the whole chip to $20.1^{\circ} \mathrm{C}$ (defined as the reference temperature).

\section{STUDY OF OPTICAL BISTABILITY}

The thermal wavelength shift of resonances is jointly determined by net heat flow (NHF) and conducted heat flow (CHF) [60]. Specifically, when the resonance wavelength gets closer to the pump, an increasing power is coupled into the ring cavity, which promotes NHF, enlarges the refractive index, and redshifts the resonance. Simultaneously, net heat in the SOI chip is conducted to the environment and blueshifts the resonance, where CHF becomes larger with an increasing temperature difference between the chip and its environment. The thermal stabilization is built when CHF balances with NHF, which can take place at both the on-resonance and the off-resonance conditions. Here, we study the optical bistability with respect to the pair rate in the nonclassical single-photon regime and employ the corresponding normalized transmittance, demonstrated in a number of studies [54-60], as a comparison. Through the reverse thinking of the working principle, we tune the resonance to match the fixed pump by varying the external temperature of the temperaturecontrolling module, which ensures that the on-resonance at different power levels can be achieved at the same wavelength. Therefore, the external temperature response in Fig. 2 behaves in an opposite way compared to the pump wavelength response in Ref. [60].

According to recent studies, the pair rate is quadratic in the circling power

$$
P_{c}=P_{p}\left|F\left(\omega_{p}\right)\right|^{2} \propto \frac{P_{p}}{\left(\Delta \omega_{\mathrm{pr}} / \omega_{\mathrm{res}}\right)^{2}+1 / Q^{2}},
$$

where $P_{p}$ denotes the incident power in the bus waveguide, $F(\omega)$ denotes the enhancement factor $[44,62]$, and $\Delta \omega_{\mathrm{pr}}=$ $\left|\omega_{p}-\omega_{\text {res }}\right|$ denotes the pump-resonance detuning, respectively. Thus, in the cooling process, where the resonance wavelength becomes shorter and gets closer to the pump, the pair rate increases with the pump-resonance detuning reduction (which behaves like the normalized transmittance reduction). The net heat starts accumulating in the ring cavity and redshifts the resonance against the blueshift effect of the active cooling. At the critical condition, where the pump is exactly on-resonance, the pair rate reaches the maximum; meanwhile, the normalized transmittance reaches the minimum. Afterward, the reducing circling power forms a synergy with the active cooling and quickly draws both the pair rate and the normalized transmittance back to the initial level [see Figs. 2(a) and 2(c)]. Note that a large temperature difference between the chip and its environment is required, so that CHF can balance with 


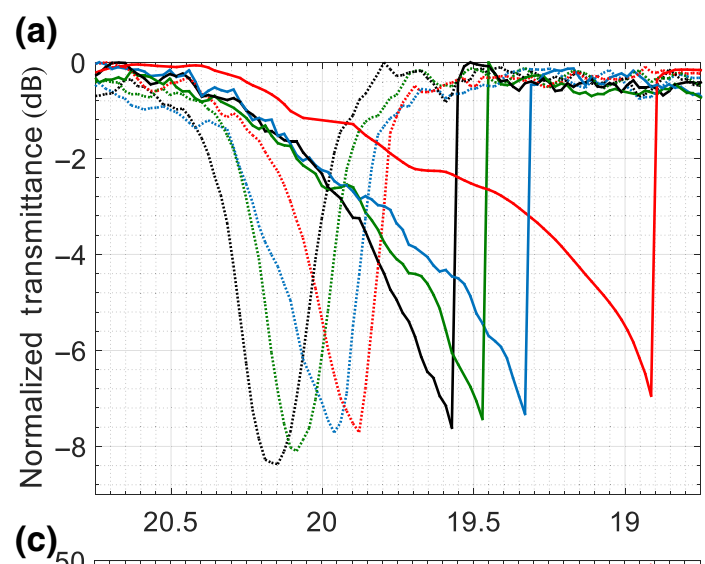

(b)
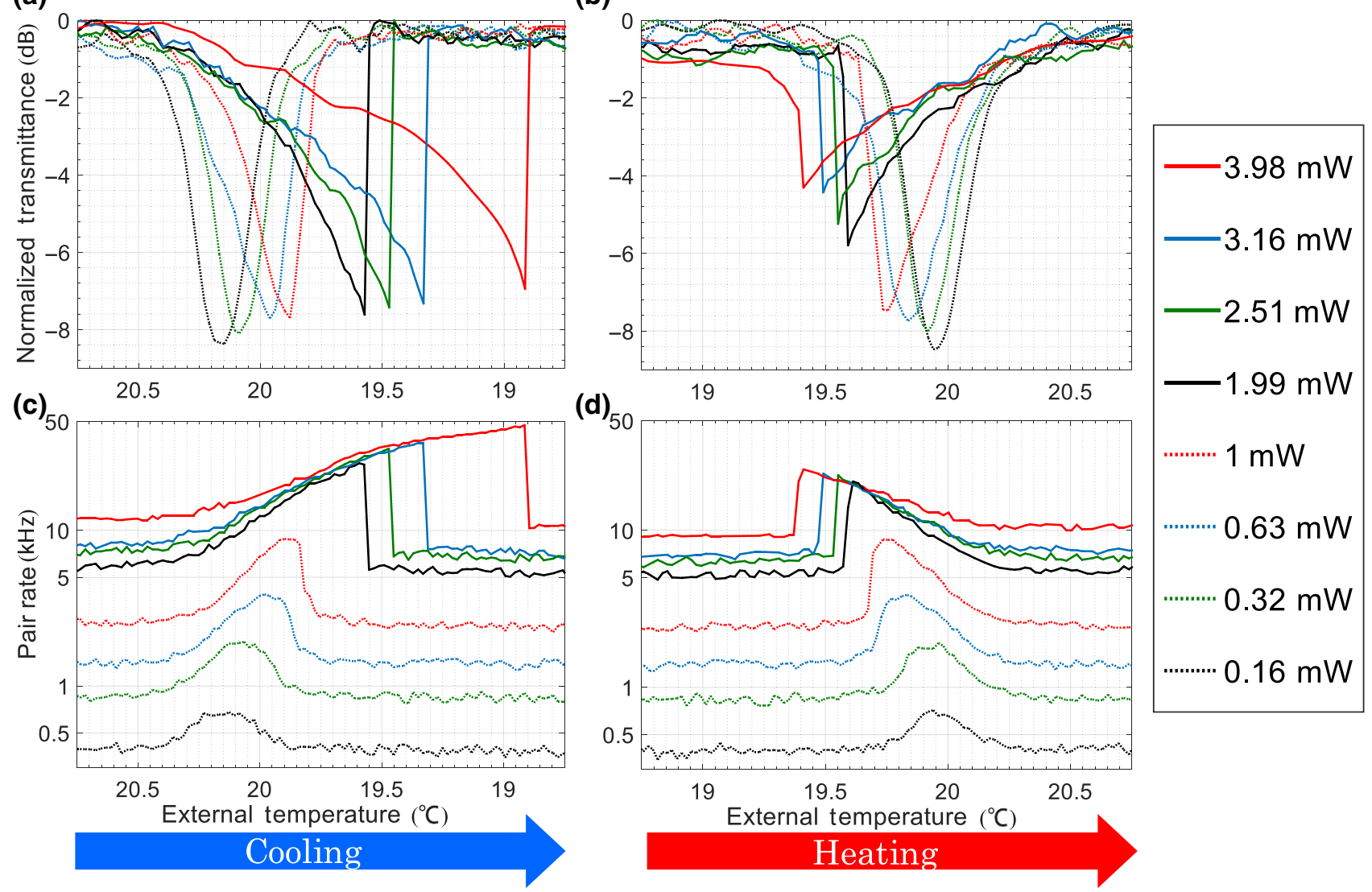

FIG. 2. The measured normalized transmittance versus the external temperature in the cooling process (a) and heating process (b), and the corresponding pair rate versus the external temperature in the cooling process (c) and heating process (d). The incident powers in the bus waveguide are $3.98 \mathrm{~mW}$ (red solid), $3.16 \mathrm{~mW}$ (blue solid), $2.51 \mathrm{~mW}$ (green solid), $1.99 \mathrm{~mW}$ (black solid), $1 \mathrm{~mW}$ (red dotted), $0.63 \mathrm{~mW}$ (blue dotted), $0.32 \mathrm{~mW}$ (green dotted), and $0.16 \mathrm{~mW}$ (black dotted), respectively.

NHF. Moreover, in the heating process, the foregoing resonance wavelength becomes longer and gets closer to the pump. The increasing circling power accelerates the active heating and quickly draws the pair rate to the maximum. Note that from theoretical study [60], the minimal normalized transmittance takes the same value in heating and cooling processes, yet our equipment fails to record such a drop-rise moment, especially at high power. After the critical condition, the pair rate and the normalized transmittances reach the initial level [see Figs. 2(b) and 2(d)]. Additionally, the on-resonance achieved by active cooling behaves more stably than that achieved by active heating, which validates the equilibrium solution of thermal dynamic equations [60].

Although the optical bistability with respect to power in the classical regime is well known and it is easy to derive a similar property in the nonclassical regime, the hysteresis behavior of the pair rate provides an explicit experimental validation. Such an understanding is further strengthened by comparing the hysteresis behavior of the pair rate to that of the normalized transmittance at a fixed external temperature of $18.8^{\circ} \mathrm{C}$ (see Fig. 3). Since it remains a challenge to keep the identical conditions of repeated experiments due to the undetectable heat accumulation inside the ring resonator, we extract a set of data through a very quick measurement. By turning up the input power, the pair rate increases slowly with a value of less than $10 \mathrm{kHz}$, which reveals the off-resonance stabilization. The pair rate dramatically rises to over $40 \mathrm{kHz}$, when the input power reaches $3.8 \mathrm{~mW}$, demonstrating the on-resonance stabilization. Similar behavior takes place by turning down the input power, in which the switch between two types of stabilization occurs at $3.4 \mathrm{~mW}[55,56,60]$.

The output power is linear to the input power in both of the stable states, the off-resonance at low power and the on-resonance at high power. The normalized transmittance remains almost unchanged in two stable states, which takes a higher value at the off-resonance (e.g., 0.23 at $3 \mathrm{~mW}$ ) and a lower value at the on-resonance (e.g., 0.06 at $4 \mathrm{~mW}$ ). According to Eq. (2), the circling power is also linear to the input power; thus, the pair rate increases with the increasing input power in both stable states. Since the enhancement factor at the on-resonance $(\Delta \omega=0)$ is much higher than that at the off-resonance $(\Delta \omega \neq 0)$, the 

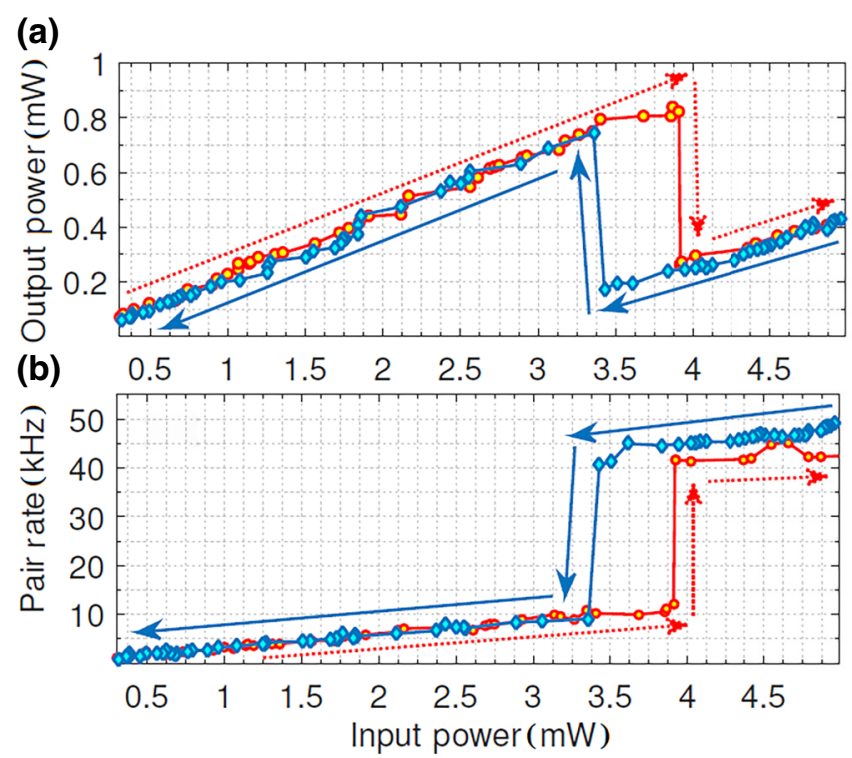

FIG. 3. (a) The output power and (b) the pair rate versus the input power, with an external temperature of $18.8^{\circ} \mathrm{C}$.

resulting pair rate runs in different levels. Note that the normalized transmittance at high power after switching cannot be extrapolated to zero, where $P_{\text {out }}-P_{\text {in }}$ curves in two stable states seem to run parallel. Such an issue takes place because the linewidth of the pump laser is wider than the FWHM of the resonance; that is, a part of the pump field always passes through the bus waveguide directly even at the on-resonance. On the other hand, the free-carrier absorption in the ring cavity increases dramatically at high power, which greatly reduces the intrinsic quality factor and the extinction ratio [62]. Hence, the normalized transmittance remains increasing, while the resulting pair rate starts saturating with the increasing input power.

Although the above optical bistability characterization is carried out in a continuous-wave-pumped photon-pair source, it also remains valid in pulsed-pumped sources with the same average power. Being experimentally proved, the strict correspondence between pair rate and output power enlightens a variety of potential functionalities in the single-photon regime using (and not limited to thermal-based) optical bistability.

\section{RESONANCE-LOCKED PHOTON-PAIR SOURCE}

For efficient and stable photon-pair generation using MRR, it remains a challenge to keep the on-resonance.
Previous studies often tune the pump to match the resonance, yet the on-resonance stabilization at different levels is built at different wavelengths [43-48,60,63]. While high-resolution tunable pumps are needed to drive these sources, the wavelength of the emitting photons heavily depends on the environmental temperature and the input power. More significantly, when the initial resonance detunes with the devices in communication systems, temperature controlling becomes necessary to achieve the wavelength matching. Hence, under the same principle of balancing CHF and NHF [54], we propose a resonancelocked method. By cooling the chip, the closest resonance is blue-tuned toward the pump and the external temperature corresponding to the critical condition is obtained. The controlling current is carefully adjusted until the onresonance stabilization is built at different power levels (see Table I). Note that in other on-resonance strategies by counteracting the power- or free-carrier-induced phase shift, a given power or a special MRR design is required $[64,65]$. As a comparison, this simple method is proved to be efficient in our setup for long-time measurements (more than an hour) and is worth popularizing in future works, where different initial resonances of various MRR samples can match the given wavelengths of the systems.

To validate the proposed resonance-locked regime, we make a long-term stable measurement with respect to the raw coincidence counts $N_{\mathrm{cc}}$ at a pump power of $4.86 \mathrm{~mW}$. Table II shows the critical temperature, the corresponding controlling current, and the raw coincidence counts at room temperatures of $20^{\circ} \mathrm{C}, 22^{\circ} \mathrm{C}$ and $24^{\circ} \mathrm{C}$, respectively. By manually adjusting the controlling current two or three times, we keep the on-resonance over $12 \mathrm{~h}$ at each room temperature. Note that the experiment is carried out in an open environment; that is, the room temperature is simply controlled by the air conditioner of the lab. Hence, the stable measurement proves that the proposed resonance-locked regime is of great potential in real-world applications. The issue of manual adjustment can be, in future works, omitted by introducing a feedback mechanism into the temperature-controlling module.

Under the on-resonance condition (room temperature of $22^{\circ} \mathrm{C}$ ), we measure the raw coincidence counts $N_{\text {cc }}$, counting the average coincidence events in the true coincidence window of $405 \mathrm{ps}$, and the accidental coincidence counts $N_{\text {acc }}$, counting the average coincidence events in the temporal window of $81 \mathrm{~ns} . N_{\mathrm{cc}}$ is proportional to pair rate $N_{c}$ and quadratic in power, which is validated as the fitted

TABLE I. The critical temperature and the corresponding controlling current for the thermal-locked regime, at $22{ }^{\circ} \mathrm{C}$.

\begin{tabular}{lcccccccccccc}
\hline \hline$P_{p}(\mathrm{~mW})$ & 0.16 & 0.32 & 0.63 & 0.80 & 0.99 & 1.26 & 1.58 & 1.99 & 2.51 & 3.16 & 3.98 & 4.86 \\
\hline$T_{c}\left({ }^{\circ} \mathrm{C}\right)$ & 20.19 & 20.11 & 19.98 & 19.90 & 19.82 & 19.74 & 19.67 & 19.58 & 19.49 & 19.35 & 18.93 & 18.31 \\
Current $(\mathrm{mA})$ & 232 & 235 & 237 & 238 & 240 & 244 & 250 & 255 & 265 & 275 & 302 & 336 \\
\hline \hline
\end{tabular}


TABLE II. The critical temperature, the controlling current, and the raw coincidence counts for stable measurement.

\begin{tabular}{lccc}
\hline \hline Room temperature & $20{ }^{\circ} \mathrm{C}$ & $22{ }^{\circ} \mathrm{C}$ & $24{ }^{\circ} \mathrm{C}$ \\
\hline Critical temperature $\left({ }^{\circ} \mathrm{C}\right)$ & 18.58 & 18.31 & 17.85 \\
Controlling current $(\mathrm{mA})$ & 232 & 235 & 237 \\
Maximal $N_{\mathrm{cc}}\left(\mathrm{min}^{-1}\right)$ & 1237 & 1248 & 1231 \\
Minimal $N_{\mathrm{cc}}\left(\mathrm{min}^{-1}\right)$ & 1159 & 1163 & 1151 \\
\hline \hline
\end{tabular}

slope of $N_{\mathrm{cc}}$ versus $P_{p}$ in the $\log$-log scale reaches 1.91 , by taking the data at low power $\left(P_{p}<2 \mathrm{~mW}\right)$ into account (see Fig. 4). However, the fitted slope reduces to 1.53 for all data, revealing the pair rate saturation induced by the avalanche detector and nonlinear loss. On one hand, the modified pair rate omitting detector saturation is given by

$$
N_{\mathrm{mc}}=\frac{N_{c}-d}{1-N_{c} \tau_{d}}
$$

where $d$ and $\tau_{d}$ denote the dark count rate and dead time, respectively [44]. On the other hand, the total quality factor in Eq. (1) also satisfies

$$
\frac{1}{Q}=\frac{1}{Q_{e}}+\frac{1}{Q_{i}}
$$

where $Q_{i}$ and $Q_{e}$ are the intrinsic quality factor and the external quality factor, respectively. Here, $Q_{e}$, depending on the bus-ring coupling coefficient, remains unchanged, while $Q_{i}$, determined by the round-trip loss, becomes lower with increasing power-dependent nonlinear loss $[44,66]$.

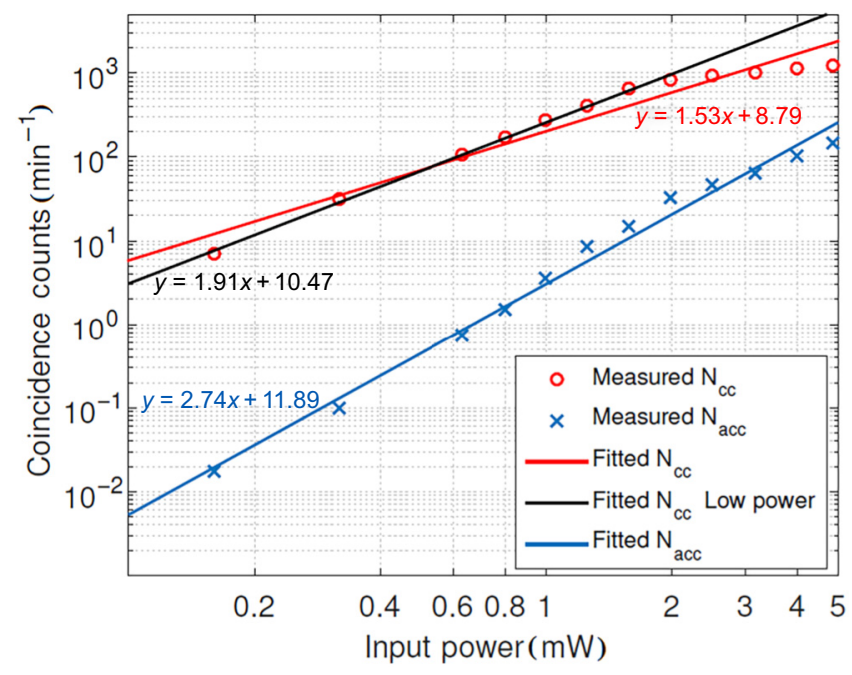

FIG. 4. The measured raw coincidence counts $N_{\text {cc }}$ (red circle) and accidental coincidence counts $N_{\text {acc }}$ versus input power per minute. The fitted slope of $N_{\mathrm{cc}}$ at all powers, $N_{\mathrm{cc}}$ at low powers, and $N_{\text {acc }}$ at all powers are $1.53,1.91$, and 2.74 , respectively.
This is also presented in Fig. 2, where the extinction ratio of the minimal to maximal normalized transmittance,

$$
\Gamma=\left|\frac{Q_{e}-Q_{i}}{Q_{e}+Q_{i}}\right|^{2},
$$

becomes larger at high power. By combining Eqs. (1), (4), and (5), the initial $Q_{e}$ and $Q_{i}$ are estimated at $8.52 \times$ $10^{4}$ and $6.95 \times 10^{4}$, respectively. At $P_{p}=4.86 \mathrm{~mW}, Q_{i}$ reduces to $6.95 \times 10^{4}$ and results in $Q=5.13 \times 10^{4}$. From Eq. (3) and $N_{\mathrm{mc}} \propto Q^{7}[44,62]$, the highest $N_{\mathrm{cc}}$ of $1232 \pm$ $20 \mathrm{~min}^{-1}$ is modified to $7787 \pm 126 \mathrm{~min}^{-1}$, which agrees with the quadratic dependence better. Moreover, the measured pair brightness, given by

$$
B_{m}=\frac{N_{\mathrm{cc}}}{\Delta \lambda P_{p}}
$$

at $1 \mathrm{~mW}$ reaches $176 \pm 40(\mathrm{~s} \mathrm{~mW} \mathrm{~nm})^{-1}$. Note that Eq. (6) uses the measured coincidence rate $N_{\mathrm{cc}}$ and the incident power in the bus waveguide, instead of the calculated pair rate and the coupled power in the ring cavity, respectively. Hence, the measured pair brightness is naturally far lower than that given in the previous studies using the internal pair brightness definition. The total loss through the afterchip setup is $21 \mathrm{~dB}$, including the coupling loss of the output PCGC, the insertion loss of the fiber-based components in the collection schemes, and the detection loss of SPDs. By omitting all loss mechanisms, the expected coincidence rate and pair brightness can be 4 orders of magnitude higher. Hence, the internal pair brightness at $1 \mathrm{~mW}$ reaches $2.82 \pm 0.65 \times 10^{6}(\mathrm{~s} \mathrm{~mW} \mathrm{~nm})^{-1}$, which approaches the results of MRR-based sources in previous works [43-46] and is an order of magnitude higher than those using strip or slow-light enhanced waveguides $[16,67,68]$.

The accidental coincidence counts follow $N_{\text {acc }} \propto\left(N_{c}+\right.$ $\left.N_{n}+d / \eta\right)^{2}$, where $N_{n}$ represents the photon contribution from the pump sideband noise, leaked pump field, and spontaneous Raman scattering. The collection efficiency $\eta$ consists of the coupling efficiency of the output PCGC, the transmittance of the after-chip filters, and the detection efficiency of SPDs. As $N_{c}$ is quadratic in power, $N_{n}$ is linear in power, and $d$ is constant, $N_{\text {acc }}$ has a fourthorder polynomial dependence on power. The fitted slope of 2.74 indicates that the contribution of $N_{c}$ is dominant; meanwhile, the measured $N_{\text {acc }}$ of $144 \pm 2 \mathrm{~min}^{-1}$ at $P_{p}=$ $4.86 \mathrm{~mW}$ can be further modified to $5788 \pm 95 \mathrm{~min}^{-1}$, which agrees well with the biquadratic dependence. Moreover, $N_{\mathrm{cc}}$ is $1-3$ orders of magnitude higher than $N_{\mathrm{acc}}$, directly illustrating efficient noise suppression [9]. The coincidence peak, resolved in a measurement time window of $405 \mathrm{ps}$ (wider than the SPD's jitter time of $300 \mathrm{ps}$ ), is far higher than the background. The measured CAR given 


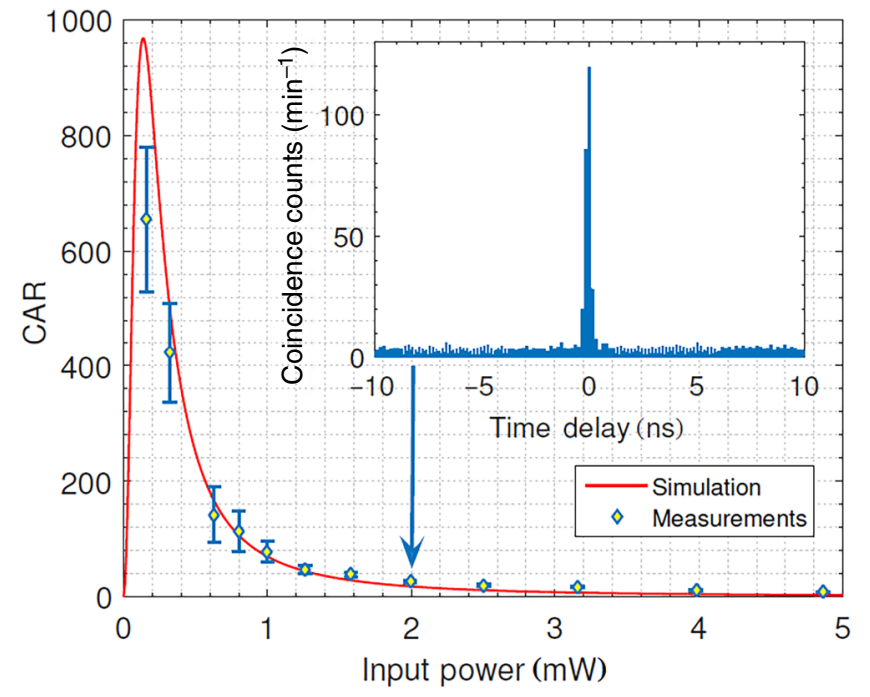

FIG. 5. The CAR versus the input power. The inset is the temporal histogram at $P_{p}=1.99 \mathrm{~mW}$.

by

$$
\mathrm{CAR}=\frac{N_{\mathrm{cc}}-N_{\mathrm{acc}}}{N_{\mathrm{acc}}}
$$

increases with decreasing input power and the highest value of $654 \pm 125$ takes place at $P_{p}=0.16 \mathrm{~mW}$, yet the coincidence rate is only $0.13 \pm 0.04 \mathrm{~Hz}$ (see Fig. 5). The highest coincidence rate of $20.53 \pm 0.34 \mathrm{~Hz}$ corresponds to a CAR of $9 \pm 0.2$, which remains in the low-noise regime.

To ensure that the photon-pair source operates in the single-photon regime, we measure the heralded secondorder correlation function, given by [67]

$$
g_{H}^{(2)}(\tau)=\frac{N_{H} N_{H A B}(\tau)}{N_{H A} N_{H B}(\tau)} .
$$

In detection scheme (2), the idler photons are separated into two arms $(A$ and $B$ ), while the signal photons are used for heralding $(H)$. In Eq. (8), $\tau$ denotes the temporal delay between arm $A$ and $B$, introduced by inserting or removing a certain length of optical fibers; $N_{H}$ denotes the heralded photon counts in arm $H$; and $N_{H A}, N_{H B}$, and $N_{H A B}$ denote the coincidence counts of $H-A$ and $H-B$ and the triple-coincidence counts of $H-A-B$, respectively. The zero-delayed $g_{H}^{(2)}$ is zero for heralded single photons, but the noise contribution results in a nonzero value. Figure 6 shows that $g_{H}^{(2)}(0)$ becomes smaller with decreasing input power and reaches the minimum of $0.14 \pm 0.09$ at $P_{p}=$ $1.58 \mathrm{~mW}$ (the errorbar comes from the standard error calculation of all measured data). All of the $g_{H}^{(2)}(0)$ measurements are below 0.5 , indicating that the photon pairs operating in the single-photon regime are dominant. A time delay of $25 \mathrm{~ns}$ is introduced to evaluate the noise property,

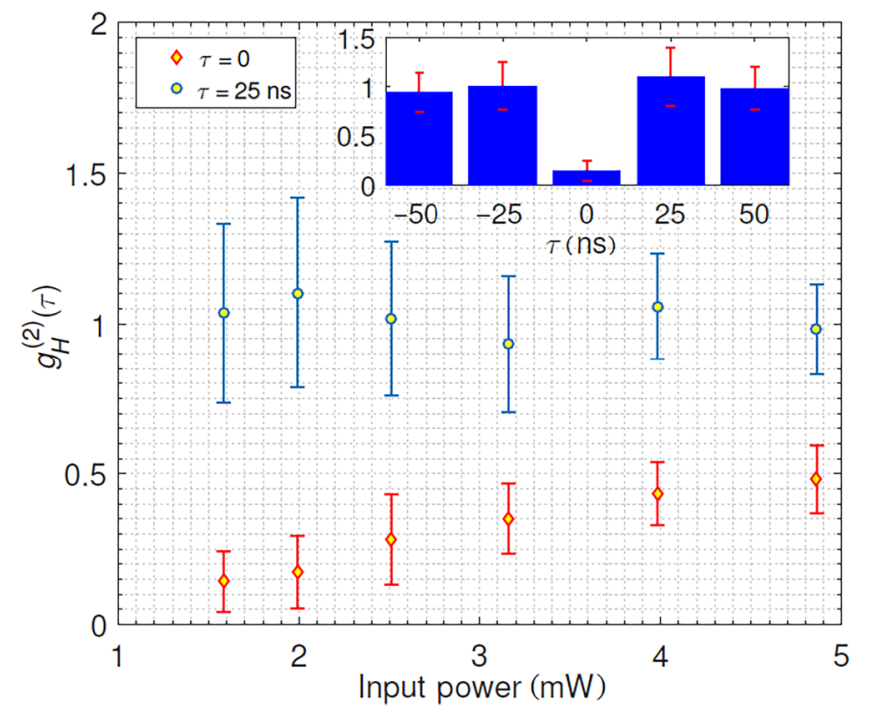

FIG. 6. The heralded second-order correlation function $g_{H}^{(2)}(\tau)$ versus input power with zero delay (red diamond) and 25-ns delay (blue circle), respectively. The inset is $g_{H}^{(2)}(\tau)$ versus the delay at $P_{p}=1.58 \mathrm{~mW}$.

where the resulting $g_{H}^{(2)}(\tau)$ varies around 1 at all powers [69]. The $g_{H}^{(2)}$ at $1.58 \mathrm{~mW}, \tau$ of $0, \pm 25 \mathrm{~ns}$, and $\pm 50 \mathrm{~ns}$ satisfies the asymptotic limit (see inset). Although the time window for searching $N_{H A B}$ is $2.5 \mathrm{~ns}$, while the included noise makes $g_{H}^{(2)}(0)$ overestimated and uncertain, no triplecoincidence events are detected at an incident power less than $1.58 \mathrm{~mW}$. Hence, these zero $g_{H}^{(2)}(0)$ are omitted from the presented data.

Through the resonance-locked method, we demonstrate stable characterization of a photon-pair source and acquire clean data. However, the figures of merit do not seem state of the art since the avalanche detectors come at the cost of relatively low speed and efficiency. This issue can, in future works, be mitigated by using the superconducting detectors with a high detection rate, low dark count, nearunity efficiency, and short deadtime [70,71]. Moreover, as nonlinear loss significantly reduces the quality factor of MRR at high power, further pair rate scaling can be achieved by using the $p-i-n$ structure valid in shorting the free carrier lifetime $[42,72]$ or by employing other materials such as (Al,Ga)As [20] to avoid nonlinear loss. Careful design of MRR is also needed to satisfy the filteringmatched free spectral range and a suitable quality factor that maximizes the enhancement factor in Eq. (2). To further increase CAR in Eq. (7), a low-loss collection scheme needs to be integrated with the photon-pair source, which emerges from the development of integrated coolers. Additionally, our resonance-locked method makes easier the on-chip integration of the pump, without requiring precision wavelength control, and the resulting photon-pair source holds great potential to support long-time operation. 


\section{CONCLUSIONS}

We experimentally validate the nonclassical optical bistability in a photon-pair source using continuous-wave pumped spontaneous four-wave mixing in an all-pass silicon-on-insulator microring resonator. We explain in detail the thermal stabilization in the on-resonance and off-resonance conditions and provide solid proof of the correspondence between classical normalized transmittance and a nonclassical pair rate, which enlightens potential applications in quantum optics. We propose a resonancelocked method using active cooling, which enables uncertain initial resonance to match the given wavelength of systems, in addition to support stable photon-pair emission. Thanks to the proposed resonance-locked regime, we stably characterize the photon-pair source and achieve clean figures of merit. The maximal coincidence rate reaches $20.53 \pm 0.34 \mathrm{~Hz}$ at the maximal pump power of $4.86 \mathrm{~mW}$. The maximal coincidental-to-accidental ratio reaches $654 \pm 125$ at a pump power of $0.16 \mathrm{~mW}$, which is several times higher than the previous studies with the same measurement time window [44]. The measurable minimal zero-delayed heralded second-order correlation is $0.14 \pm 0.09$ at a pump power of $1.58 \mathrm{~mW}$, demonstrating that our photon-pair source operates in the low-noise single-photon regime. We finally propose further pair rate scaling to focus on solving detector saturation, avoiding nonlinear loss, and fully integrating the photon-pair source on-chip.

\section{ACKNOWLEDGMENTS}

This work is jointly supported by the China Scholarship Council (CSC); National Natural Science Foundation of China (Grant No. 61671455); and Danish National Research Foundation (Grant No. DNRF123). The authors also greatly thank Yuhong Liu, Jianfa Zhang, Wei Liu, Sen Zhang, Jesper B. Christensen, Erik N. Christensen, Davide Bacco, Yunhong Ding, and Karsten Rottwitt for their support.

[1] E. Knill, R. Laflamme, and G. J. Milburn, A scheme for efficient quantum computation with linear optics, Nature 409 , 46 (2001).

[2] N. Gisin and R. Thew, Quantum communication, Nat. Photonics 1, 165 (2007).

[3] A. K. Ekert, Quantum Cryptography based on Bell's Theorem, Phys. Rev. Lett. 67, 661 (1991).

[4] C. H. Bennett, G. Brassard, and N. D. Mermin, Quantum Cryptography without Bell's Theorem, Phys. Rev. Lett. 68, 557 (1992).

[5] I. Ali-Khan, C. J. Broadbent, and J. C. Howell, Largealphabet Quantum Key Distribution using Energy-time Entangled Bipartite States, Phys. Rev. Lett. 98, 060503 (2007).
[6] P. G. Kwiat, K. Mattle, H. Weinfurter, A. Zeilinger, A. V. Sergienko, and Y. Shih, New High-intensity Source of Polarization-entangled Photon Pairs, Phys. Rev. Lett. 75, 4337 (1995).

[7] S. Tanzilli, H. De Riedmatten, H. Tittel, H. Zbinden, P. Baldi, M. De Micheli, D. B. Ostrowsky, and N. Gisin, Highly efficient photon-pair source using periodically poled lithium niobate waveguide, Electron. Lett. 37, 26 (2001).

[8] H. Takesue and K. Inoue, $1.5-\mu \mathrm{m}$ band quantum-correlated photon pair generation in dispersion-shifted fiber: Suppression of noise photons by cooling fiber, Opt. Express 13, 7832 (2005).

[9] S. D. Dyer, B. Baek, and S. W. Nam, High-brightness, lownoise, all-fiber photon pair source, Opt. Express 17, 10290 (2009).

[10] Z. Y. Zhou, Y. H. Li, L. X. Xu, B. S. Shi, and G. C. Guo, Multiplexed entangled photon-pair sources for all-fiber quantum networks, Phys. Rev. A 94, 1002904 (2016).

[11] Y. Li, Z.-Y. Zhou, D.-S. Ding, and B.-S. Shi, Cw-pumped telecom band polarization entangled photon pair generation in a sagnac interferometer, Opt. Express 23, 28792 (2015).

[12] J. E. Sharping, K. F. Lee, M. A. Foster, A. C. Turner, B. S. Schmidt, M. Lipson, A. L. Gaeta, and P. Kumar, Generation of correlated photons in nanoscale silicon waveguides, Opt. Express 14, 12388 (2006).

[13] K.-I. Harada, H. Takesue, H. Fukuda, T. Tsuchizawa, T. Watanabe, K. Yamada, Y. Tokura, and S.-I. Itabashi, Frequency and polarization characteristics of correlated photon-pair generation using a silicon wire waveguide, IEEE J. Sel. Top. Quantum. Electron. 16, 325 (2010).

[14] Y.-H. Li, Z.-Y. Zhou, L.-T. Feng, W.-T. Fang, S.-L. Liu, S.K. Liu, K. Wang, X.-F. Ren, D.-S. Ding, L.-X. Xu et al., On-chip Multiplexed Multiple Entanglement Sources in a Single Silicon Nanowire, Phys. Rev. Appl. 7, 064005 (2017).

[15] W.-T. Fang, Y.-H. Li, Z.-Y. Zhou, L.-X. Xu, G.-C. Guo, and B.-S. Shi, On-chip generation of time-and wavelengthdivision multiplexed multiple time-bin entanglement, Opt. Express 26, 12912 (2018).

[16] K. Guo, E. N. Christensen, J. B. Christensen, J. G. Koefoed, D. Bacco, Y. Ding, H. Ou, and K. Rottwitt, High coincidence-to-accidental ratio continuous-wave photonpair generation in a grating-coupled silicon strip waveguide, Appl. Phys. Express 10, 062801 (2017).

[17] S. Clemmen, A. Perret, S. Selvaraja, W. Bogaerts, D. Van Thourhout, R. Baets, P. Emplit, and S. Massar, Generation of correlated photons in hydrogenated amorphous-silicon waveguides, Opt. Lett. 35, 3483 (2010).

[18] J. B. Spring, P. S. Salter, B. J. Metcalf, P. C. Humphreys, M. Moore, N. Thomas-Peter, M. Barbieri, X.-M. Jin, N. K. Langford, W. S. Kolthammer, M. J. Booth, and A. W. Ian, On-chip low loss heralded source of pure single photons, Opt. Express 21, 13522 (2013).

[19] C. Xiong, X. Zhang, A. Mahendra, J. He, D.-Y. Choi, C. Chae, D. Marpaung, A. Leinse, R. Heideman, M. Hoekman, C. G. H. Roeloffzen, R. M. Oldenbeuving, P. W. L. van Dijk, C. Taddei, P. H. W. Leong, and B. J. Eggleton, Compact and reconfigurable silicon nitride time-bin entanglement circuit, Optica 2, 724 (2015). 
[20] P. Kultavewuti, E. Y. Zhu, L. Qian, V. Pusino, M. Sorel, and J. S. Aitchison, Correlated photon pair generation in $\mathrm{AlGaAs}$ nanowaveguides via spontaneous four-wave mixing, Opt. Express 24, 3365 (2016).

[21] N. C. Harris, D. Grassani, A. Simbula, M. Pant, M. Galli, T. Baehr-Jones, M. Hochberg, D. Englund, D. Bajoni, and C. Galland, Integrated Source of Spectrally Filtered Correlated Photons for Large-scale Quantum Photonic Systems, Phys. Rev. X 4, 041047 (2014).

[22] J. Wang, D. Bonneau, M. Villa, J. W. Silverstone, R. Santagati, S. Miki, T. Yamashita, M. Fujiwara, M. Sasaki, H. Terai, M. G. Tanner, C. M. Natarajan, R. H. Hadfield, Jeremy L. O'Brien, and M. G. Thompson, Chip-to-chip quantum photonic interconnect bypath-polarization interconversion, Optica 3, 407 (2016).

[23] R. Soref, The past, present, and future of silicon photonics, IEEE J. Sel. Top. Quantum Electron. 12, 1678 (2006).

[24] J. Leuthold, C. Koos, and W. Freude, Nonlinear silicon photonics, Nat. Photonics 4, 535 (2010).

[25] T. Liang and H. Tsang, Efficient raman amplification in silicon-on-insulator wave guides, Appl. Phys. Lett. 85, 3343 (2004).

[26] M. Pu, L. Liu, H. Ou, K. Yvind, and J. M. Hvam, Ultra-lowloss inverted taper coupler for silicon-on-insulator ridge waveguide, Opt. Commun. 283, 3678 (2010).

[27] Y. Ding, H. Ou, and C. Peucheret, Ultrahigh-efficiency apodized grating coupler using fully etched photonic crystals, Opt. Lett. 38, 2732 (2013).

[28] W. Tittel, J. Brendel, N. Gisin, and H. Zbinden, Longdistance bell-type tests using energy-time entangled photons, Phys. Rev. A 59, 4150 (1999).

[29] Q. Zhang, H. Takesue, S. W. Nam, C. Langrock, X. Xie, B. Baek, M. M. Fejer, and Y. Yamamoto, Distribution of time-energy entanglement over $100 \mathrm{~km}$ fiber using superconducting single photon detectors, Opt. Express 16, 5776 (2008).

[30] S. Dong, Q. Zhou, W. Zhang, Y. He, W. Zhang, L. You, Y. Huang, and J. Peng, Energy-time entanglement generation in optical fibers under CW pumping, Opt. Express 22, 359 (2014).

[31] S. Dong, L. Yu, W. Zhang, J. Wu, W. Zhang, L. You, and Y. Huang, Generation of hyper-entanglement in polarization/energy-time and discrete-frequency/energytime in optical fibers, Sci. Rep. 5, 9195 (2015).

[32] C. Reimer, L. Caspani, M. Clerici, M. Ferrera, M. Kues, M. Peccianti, A. Pasquazi, L. Razzari, B. E. Little, S. T. Chu, D. J. Moss, and R. Morandotti, Integrated frequency comb source of heralded single photons, Opt. Express 22, 6535 (2014).

[33] F. Mazeas, M. Traetta, M. Bentivegna, F. Kaiser, D. Aktas, W. Zhang, C. Ramos, L. Ngah, T. Lunghi, E. Picholle et al., High-quality photonic entanglement for wavelengthmultiplexed quantum communication based on a silicon chip, Opt. Express 24, 28731 (2016).

[34] S. Wengerowsky, S. K. Joshi, F. Steinlechner, H. Hübel, and R. Ursin, Entanglement-based wavelength multiplexed quantum communication network, arXiv:1801.06194 (2018).

[35] J. M. Arrazola and V. Scarani, Covert Quantum Communication, Phys. Rev. Lett. 117, 250503 (2016).
[36] M. Davanco, J. R. Ong, A. B. Shehata, A. Tosi, I. Agha, S. Assefa, F. Xia, W. M. Green, S. Mookherjea, and K. Srinivasan, Telecommunications-band heralded single photons from a silicon nanophotonic chip, Appl. Phys. Lett. 100, 261104 (2012).

[37] I. A. Walmsley and M. G. Raymer, Toward quantuminformation processing with photons, Science 307, 1733 (2005).

[38] K. N. Cassemiro, K. Laiho, and C. Silberhorn, Accessing the purity of a single photon by the width of the hong-ou-mandel interference, New J. Phys. 12, 113052 (2010).

[39] L. Helt, Z. Yang, M. Liscidini, and J. Sipe, Spontaneous four-wave mixing in microring resonators, Opt. Lett. 35, 3006 (2010).

[40] S. Azzini, D. Grassani, M. J. Strain, M. Sorel, L. Helt, J. Sipe, M. Liscidini, M. Galli, and D. Bajoni, Ultra-low power generation of twin photons in a compact silicon ring resonator, Opt. Express 20, 23100 (2012).

[41] K.-Y. Wang, V. G. Velev, K. F. Lee, A. S. Kowligy, P. Kumar, M. A. Foster, A. C. Foster, and Y.-P. Huang, Multichannel photon-pair generation using hydrogenated amorphous silicon waveguides, Opt. Lett. 39, 914 (2014).

[42] E. Engin, D. Bonneau, C. M. Natarajan, A. S. Clark, M. Tanner, R. Hadfield, S. N. Dorenbos, V. Zwiller, K. Ohira, $\mathrm{N}$. Suzuki et al., Photon pair generation in a silicon microring resonator with reverse bias enhancement, Opt. Express 21, 27826 (2013).

[43] Y. Guo, W. Zhang, S. Dong, Y. Huang, and J. Peng, Telecom-band degenerate-frequency photon pair generation in silicon microring cavities, Opt. Lett. 39, 2526 (2014).

[44] Y. Guo, W. Zhang, N. Lv, Q. Zhou, Y. Huang, and J. Peng, The impact of nonlinear losses in the silicon micro-ring cavities on $\mathrm{CW}$ pumping correlated photon pair generation, Opt. Express 22, 2620 (2014).

[45] D. Grassani, S. Azzini, M. Liscidini, M. Galli, M. J. Strain, M. Sorel, J. Sipe, and D. Bajoni, Micrometer-scale integrated silicon source of time-energy entangled photons, Optica 2, 88 (2015).

[46] R. Wakabayashi, M. Fujiwara, K.-I. Yoshino, Y. Nambu, M. Sasaki, and T. Aoki, Time-bin entangled photon pair generation from Si micro-ring resonator, Opt. Express 23, 1103 (2015).

[47] S. Clemmen, K. P. Huy, W. Bogaerts, R. G. Baets, P. Emplit, and $\mathrm{S}$. Massar, Continuous wave photon pair generation in silicon-on-insulator waveguides and ring resonators, Opt. Express 17, 16558 (2009).

[48] E. Hemsley, D. Bonneau, J. Pelc, R. Beausoleil, J. L. O'Brien, and M. G. Thompson, Photon pair generation in hydrogenated amorphous silicon microring resonators, Sci. Rep. 6, 38908 (2016).

[49] Y.-H. Li, W.-T. Fang, Z.-Y. Zhou, S.-L. Liu, S.-K. Liu, Z.H. Xu, C. Yang, Y. Li, L.-X. Xu, G.-C. Guo et al., Quantum frequency conversion for multiplexed entangled states generated from micro-ring silicon chip, Opt. Express 26, 28429 (2018).

[50] F. Ramiro-Manzano, N. Prtljaga, L. Pavesi, G. Pucker, and M. Ghulinyan, Thermo-optical bistability with $\mathrm{Si}$ 
nanocrystals in a whispering gallery mode resonator, Opt. Lett. 38, 3562 (2013).

[51] M. Bernard, F. R. Manzano, L. Pavesi, G. Pucker, I. Carusotto, and M. Ghulinyan, Complete crossing of fano resonances in an optical microcavity via nonlinear tuning, Photonics Res. 5, 168 (2017).

[52] D. Dimitropoulos, R. Jhaveri, R. Claps, J. Woo, and B. Jalali, Lifetime of photogenerated carriers in silicon-oninsulator rib waveguides, Appl. Phys. Lett. 86, 071115 (2005).

[53] H. Rong, A. Liu, R. Nicolaescu, M. Paniccia, O. Cohen, and D. Hak, Raman gain and nonlinear optical absorption measurements in alow-loss silicon waveguide, Appl. Phys. Lett. 85, 2196 (2004).

[54] V. R. Almeida and M. Lipson, Optical bistability on a silicon chip, Opt. Lett. 29, 2387 (2004).

[55] G. Priem, P. Dumon, W. Bogaerts, D. Van Thourhout, G. Morthier, and R. Baets, Optical bistability and pulsating behaviour in silicon-on-insulator ring resonator structures, Opt. Express 13, 9623 (2005).

[56] Q. Xu and M. Lipson, Carrier-induced optical bistability in silicon ring resonators, Opt. Lett. 31, 341 (2006).

[57] X.-S. Lin, Q. Shi, X.-H. Cai, N.-S. Zhao, and S. Lan, Dynamic shift effect on bistability in nonlinear optical microcavities, Opt. Express 14, 6794 (2006).

[58] I. D. Rukhlenko, M. Premaratne, and G. P. Agrawal, Analytical study of optical bistability in silicon ring resonators, Opt. Lett. 35, 55 (2010).

[59] X. Zheng, Y. Luo, G. Li, I. Shubin, H. Thacker, J. Yao, K. Raj, J. E. Cunningham, and A. V. Krishnamoorthy, Enhanced optical bistability from self-heating due to free carrier absorption in substrate removed silicon ring modulators, Opt. Express 20, 11478 (2012).

[60] T. Carmon, L. Yang, and K. J. Vahala, Dynamical thermal behavior and thermal self-stability of microcavities, Opt. Express 12, 4742 (2004).

[61] W. Bogaerts, P. De Heyn, T. Van Vaerenbergh, K. De Vos, S. Kumar Selvaraja, T. Claes, P. Dumon, P. Bienstman, D. Van Thourhout, and R. Baets, Silicon microring resonators, Laser. Photon. Rev. 6, 47 (2012).
[62] K. Guo, X. Shi, X. Wang, J. Yang, Y. Ding, H. Ou, and Y. Zhao, Generation rate scaling: The quality factor optimization of microring resonators for photon-pair sources, Photon. Res. 6, 587 (2018).

[63] K. Padmaraju and K. Bergman, Resolving the thermal challenges for silicon microring resonator devices, Nanophotonics 3, 269 (2014).

[64] L.-W. Luo, G. S. Wiederhecker, K. Preston, and M. Lipson, Power insensitive silicon microring resonators, Opt. Lett. 37, 590 (2012).

[65] M. Notomi, T. Tanabe, A. Shinya, E. Kuramochi, H. Taniyama, S. Mitsugi, and M. Morita, Nonlinear and adiabatic control of high-q photonic crystal nanocavities, Opt. Express 15, 17458 (2007).

[66] B. E. Little, S. T. Chu, H. A. Haus, J. Foresi, and J.-P. Laine, Microring resonator channel dropping filters, Journal Light Wave Technol. 15, 998 (1997).

[67] A. S. Clark, C. Husko, M. J. Collins, G. Lehoucq, S. Xavier, A. De Rossi, S. Combrié, C. Xiong, and B. J. Eggleton, Heralded single-photon source in a III-V photonic crystal, Opt. Lett. 38, 649 (2013).

[68] C. Xiong, B. Bell, and B. J. Eggleton, Cmos-compatible photonic devices for single-photon generation, Nanophotonics 5, 427 (2016).

[69] X.-S. Ma, S. Zotter, J. Kofler, T. Jennewein, and A. Zeilinger, Experimental generation of single photons via active multiplexing, Phys. Rev. A 83, 043814 (2011).

[70] B. Korzh, N. Walenta, T. Lunghi, N. Gisin, and H. Zbinden, Free-running in gaas single photon detector with 1 dark count per second at 10\% efficiency, Appl. Phys. Lett. 104, 081108 (2014).

[71] L. C. Comandar, B. Fröhlich, J. F. Dynes, A. W. Sharpe, M. Lucamarini, Z. Yuan, R. V. Penty, and A. J. Shields, Gigahertz-gated ingaas/inp single-photon detector with detection efficiency exceeding $55 \%$ at $1550 \mathrm{~nm}$, J. Appl. Phys. 117, 083109 (2015).

[72] A. C. Turner-Foster, M. A. Foster, J. S. Levy, C. B. Poitras, R. Salem, A. L. Gaeta, and M. Lipson, Ultrashort freecarrier lifetime in low-loss silicon nanowaveguides, Opt. Express 18, 3582 (2010). 